\title{
Numerical Modeling of Transient Thermoelectric Transports in Silicon Nanowire
}

\author{
Woei-Yun Ho \\ Department of Applied Physics, National Pingtung University, Pingtung, Taiwan \\ email: wyho@mail.nptu.edu.tw
}

Keywords: Silicon nanowire, thermoelectric materials, transient transport

\begin{abstract}
Thermoelectric materials with high figure of merit (zT) are very important to heat engines in the harvesting of waste heat. Silicon nanowire have attracted considerable attention due to its enhanced zT as compared to its bulk counterpart. This paper reports the transient transport behaviors of the thermoelectric silicon nanowire (SiNW) with diameters of 50nm over a temperature range of 260-400 K. For an accurate modeling of thermoelectric effects, the COMSOL Multiphysics software program is used to solve 3D thermoelectric field equations. In this work, numerical simulations of the Seebeck effect and the Peltier effect have been implemented to understand the transient transport behaviors of SiNW. Study results indicates that the Seebeck effect exhibits a shorter relaxation time of electric flow while the Peltier effect needs more time to build up a steady heat flow.
\end{abstract}

\section{Introduction}

Recently, the global need for alternative sources of energy has revived interest in thermoelectric studies. There has been a tremendous amount of research has been reported in the searching for innovative thermoelectric materials (TEMs) [1]. The maturity of the nanotechnology and the ability to manipulate the materials at the atomic scale enables niche applications for this renewable energy sources even while conventional processes are more efficient. Thermoelectric effect is a two-way process involving a fundamental interplay between the electronic and thermal properties of a system. It enable the interconversion of thermal and electrical energy. The two primary thermoelectric effects are the Seebeck effect and the Peltier effect. The Seebeck effect devices are able to transform a temperature gradient into electrical current, and the Peltier effect describes how an electrical current can create a heat flow.

The thermoelectric devices have attracted significant research efforts to improve their thermoelectric performance. The efficiency of a thermoelectric material is characterized by a dimensionless figure of merit zT, which is defined as [1]:

$$
\mathrm{zT}=\frac{S^{2} \sigma T}{\kappa}
$$

where $\mathrm{S}$ is the Seebeck coefficient, $\sigma$ is the electrical conductivity, $\mathrm{T}$ is the temperature and $\kappa$ the thermal conductivities, respectively. The product $S^{2} \sigma$ is also known as power factor. Even though efforts to increase zT has been going on for 200 years, the zT has remained under 1. Conceptually, to obtain a high zT, both Seebeck coefficient and electrical conductivity must be large, while thermal conductivity must be suppressed so that the thermoelectric performance can be enhanced. However, in most materials, electrical and thermal conductivity are linked, and thus they cannot be tailored independently. It has been difficult to reduce thermal conductivity without significantly altering other properties. This feature imposes the fundamental problem in obtaining efficient TEMs. After four decades of research, most attention has been focused on $\mathrm{Bi}_{2} \mathrm{Te}_{3} \mathrm{TEM}$, because they are traditionally considered as the most widely used TEMs with the figure of merit $\mathrm{zT} \sim 1$ at room temperature.

Interest in thermoelectricity renewed in the 1990's with the influx of new ideas in the search for high zT materials. A general strategy guided by the fact is that the lattice thermal conductivity is the only parameter that is independent on carrier concentration. Therefore, reducing lattice thermal 
conductivity but keeping the electrons high mobility is an effective method to enhance thermoelectric efficient. Current research suggests that small band gap semiconductors might overcome this hurdle. Particularly, in 1993 a theoretical work from Hicks and Dresselhaus predicted the merits of silicon nanowire (SiNW) in having an enhanced thermoelectric properties and provided a novel route to the design of improved thermoelectric materials [2] [3] [4]. Theoretically, low dimensional structures could be very effective for suppressing the phonon contribution due to increased scattering at the boundaries, while keeping the electrical conductivity relatively high as compared to its bulk counterpart. With the development of technology for the fabrication of SiNW, it can become a promising candidate for a great enhancement of the figure of merit.

Due to the complex nature of the underlying physics of thermoelectrics, the field is rich in opportunities to yield new insight into our understanding of TEMs. However, there is a problem when we deal with such small devices. Experimentally measuring the thermal properties of nanowires is extremely difficult to handle, because non ideal factors have significant influence on low-dimensional devices. Along with the rapid growth of the nanotechnology, the simulation technique is important part before start the fabrication device. To understand the underlying physics and understand the interactions among these parameters, numerical modeling is becoming indispensable tool. Besides, numerical modeling is the most simple and direct way to design a device with excellent thermoelectric performance. In the past, the most of related studies have given the focus on behaviors of steady state, and transient behavior has not been addressed in most of these studies but focuses only on the use of nanowires in thermoelectric power generation. Therefore, this task is undertaken in a frame of numerical simulation and the objective of this work is to understand the transient thermoelectric transport in a SiNW. In this study, the COMSOL Multiphysics software program is used to simulate the transient thermoelectric transport in a SiNW.

\section{Numerical Modeling}

For a thermoelectric analysis, the Fourier heat transfer and the conversation of electric charge equations can be simultaneously coupled to produce a system of coupled thermoelectric field equations. To investigate the transient thermoelectric transport in nanostructured samples, the governing equations for a thermoelectric effect are given in the followings: Thermoelectric constitutive equations for electric current density $\mathbf{J}$ and heat current density $\mathbf{q}$ are written as:

$$
\begin{aligned}
& \mathbf{J}=\sigma \mathbf{E}-\mathrm{S} \nabla \mathrm{T}=-\sigma \nabla \mathrm{V}-\mathrm{S} \nabla \mathrm{T} \\
& \mathbf{q}=-\kappa \nabla \mathrm{T}+\Pi \mathbf{J}
\end{aligned}
$$

where $\mathbf{E}$ is electric field and $\mathrm{V}$ is electric potential, and the electric field can be written as $\mathbf{E}=-\nabla \mathrm{V}$. In physics, we have the dispersion relation, $\mathbf{D}=\varepsilon \mathbf{E}$, which accounts for the effects of free and bound charge within materials. It is necessary in using the displacement electric field $\mathbf{D}$ to deal with the dispersion for a linear response of the system. The alternative form of the conservation of electric charge equation connecting electric current $\mathbf{J}$ and electric displacement field $\mathbf{D}$ can be expressed as:

$$
\nabla \cdot\left(\mathbf{J}+\frac{\partial \boldsymbol{D}}{\partial t}\right)=0
$$

The Fourier heat transfer equation with heat source term $\mathrm{Q}$ is:

$$
\rho C_{p} \frac{\partial T}{\partial t}+\nabla \times \boldsymbol{q}=Q
$$

Finally taken together, the Fourier heat transfer and conversation of electric charge equations can be rewritten as the followings:

$$
\begin{aligned}
& \rho C_{p} \frac{\partial T}{\partial t}-\nabla\left[\left(\sigma S^{2} T+\kappa\right) \nabla T\right)-\nabla \cdot(\sigma S T \nabla V)=\sigma \nabla V \cdot \nabla V+\sigma \nabla V \cdot \nabla T \\
& \nabla \cdot(\sigma S \nabla T+\sigma \nabla V)=-\nabla \varepsilon \frac{\partial V}{\partial t}
\end{aligned}
$$

In above expressions, except those mentioned before, the material parameters use in the modeling are: $\Pi$ the Peltier coefficient, $\varepsilon$ the dielectric constant, $\rho$ the density, and $C_{p}$ thermal 
capacity at constant pressure, respectively. The dependent variables are the temperature $\mathrm{T}$, and the voltage, V. According to the Onsagar relation, $S$ and $\Pi$ satisfy the relation as $\Pi=\mathrm{ST}$.

In the simulations, the Seebeck coefficient $S$ and electric conductivity of SiNW are $245 \mathrm{uV} / \mathrm{K}$ and $5.88 \times 10^{4} \mathrm{~V} / \mathrm{m}$, respectively [5] [6] [7] [8]. As for thermal conductivity, it often depends on temperature, transverse dimension and surface roughness for nanomaterials. However, the quantitative relation between thermal conductivity and surface roughness remains unknown. Consequently, most simulations adopted temperature-independent quantity and ignored the factor of surface roughness for the sake of limitation of experimental data. Unavoidably, this will impose limitations to current thermoelectric models such as not having temperature-dependent thermoelectric coefficients. To accurately simulate thermoelectric transport in a SiNW, a curve fitted temperature-dependent thermal conductivity were used in this study. The experimental data of thermal conductivity for SiNW can be found in the open literatures [8] [9] [10] [11]. One can deduce the thermal conductivity based on the definition of thermal conductance $G, G=\kappa A / L$, where A is cross section area and $\mathrm{L}$ is length of sample. Consequently, the set of data is fitted with the third order polynomial which is available in the range of $200 \mathrm{~K} 400 \mathrm{~K}$. The fitted thermal conductivity $\kappa$ of SiNW with the fixed transverse dimension of $50 \mathrm{~nm}$ is given in the following:

$$
\kappa=2.356+0.0188 \mathrm{~T}+2.555 \times 10^{-5} \mathrm{~T}^{2}-1.372 \times 10^{-7}[\mathrm{~W} / \mathrm{mK}]
$$

The geometry of the modeled SiNW is shown in Fig. 1. The SiNW consists of a $50 \mathrm{nmx} 50 \mathrm{nmx} 2.2 \mathrm{um}$ silicon thermoelectric element and two $50 \mathrm{~nm} \times 50 \mathrm{~nm} \times 100 \mathrm{~nm}$ copper on each end of SiNW element. For an accurate modeling of thermoelectric effects, a commercially available software package COMSOL Multiphysics was used to implement a 3D finite element simulation of thermoelectric field equations.

\section{Simulation Results}

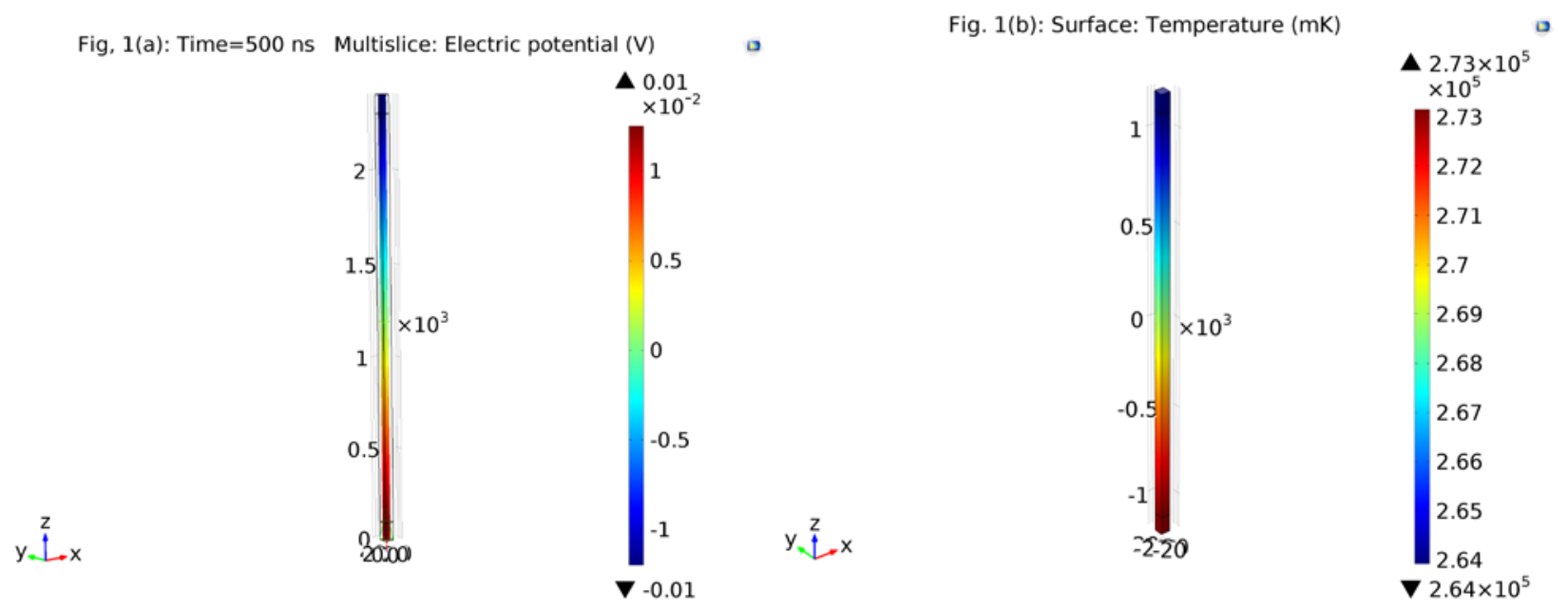

Fig. 1: (a) the build-up of electric potential profile at time 500ns in the Seebeck effect simulation, (b) the build-up of temperature profile at time 3500ns in Peltier effect simulation

In this paper, simulations of the Seebeck effect and the Peltier effect were separately implemented for an open circuit. First, numerical modeling of the steady states of the temperature and voltage were carried out. Upon a constant temperature difference applied to each end of SiNW, it results in the generation of a potential across the SiNW, as shown in Fig. 1(a), due to the Seebeck effect. The Seebeck voltage generated across the hot and cold ends is $\sim 2 \mathrm{mV}$, where hot end temperature is kept at $373 \mathrm{~K}$ and the cold end temperature is $273 \mathrm{~K}$. It is well known that space charge carriers accumulate in the each end region of SiNW resulting in the formation of an electric potential difference in the open-circuit condition. According to the Peltier effect, by maintaining a constant temperature at one end of the nanowire, a temperature difference can be created across the SiNW with an electric current, I=1.25uA, through the sample, as shown in Fig. 1(b). A temperature decrease of $9 \mathrm{~K}$ is observed.

Results shown in Fig. 2 are the transient states of temperature and electric potential profiles 
along the SiNW generated across the SiNW with varying time as indicated in the legend, respectively. Clearly, they have different relaxation times, $\tau$, or build-up times necessary for the onset of a heat flux (or electric current) after a temperature gradient (or electric potential) is imposed on a given sample. The result indicates that the time needed to reach steady state in the Seebeck effect simulation is much shorter as compared to that of Peltier effect simulation.
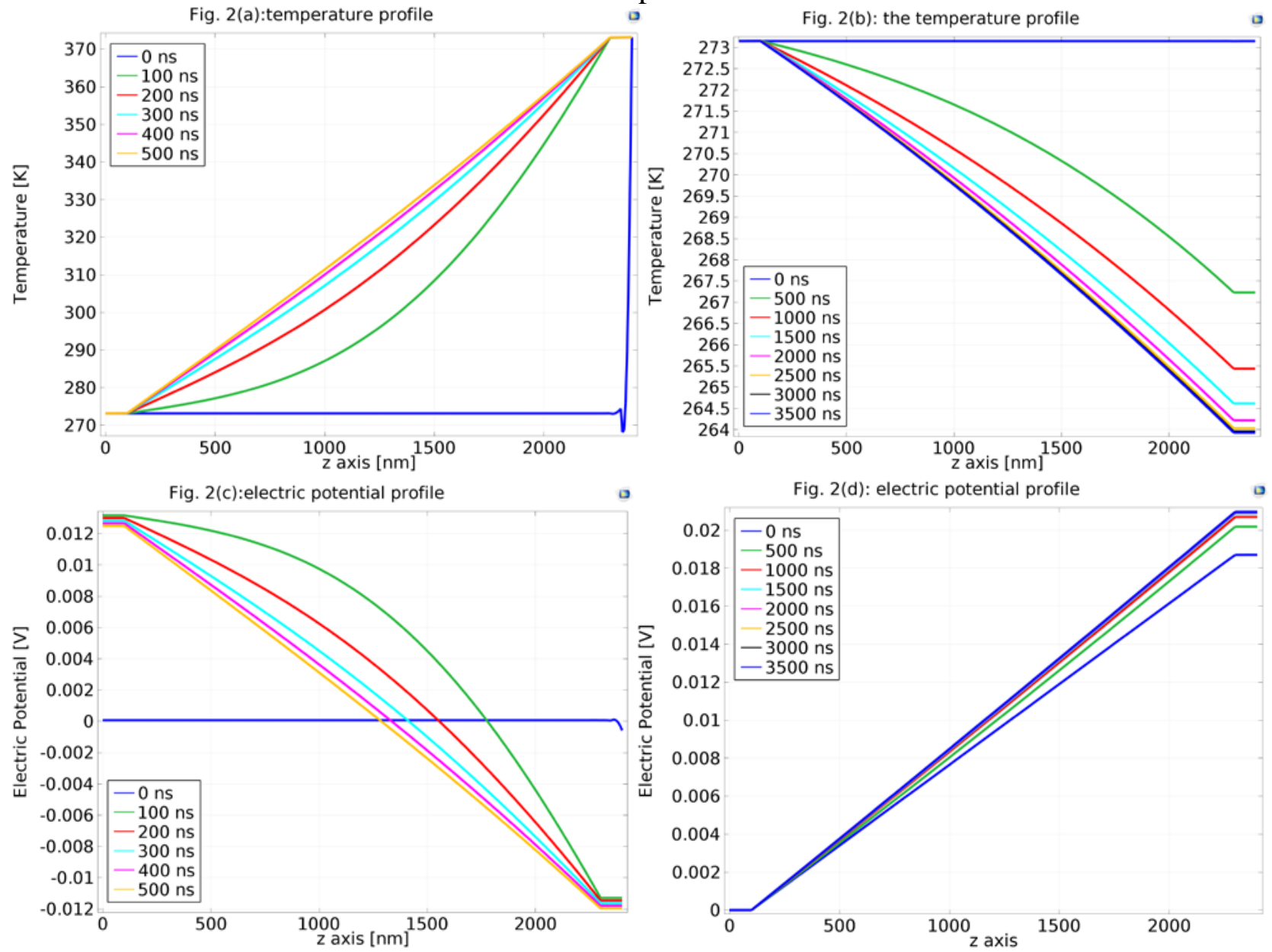

Fig. 2: (a) the temperature profile in the Seebeck effect simulation, (b) the temperature profile in the Peltier effect simulation, (c) the electric potential profile in the Seebeck simulation, (d) the electric potential profile in the Peltier effect simulation.
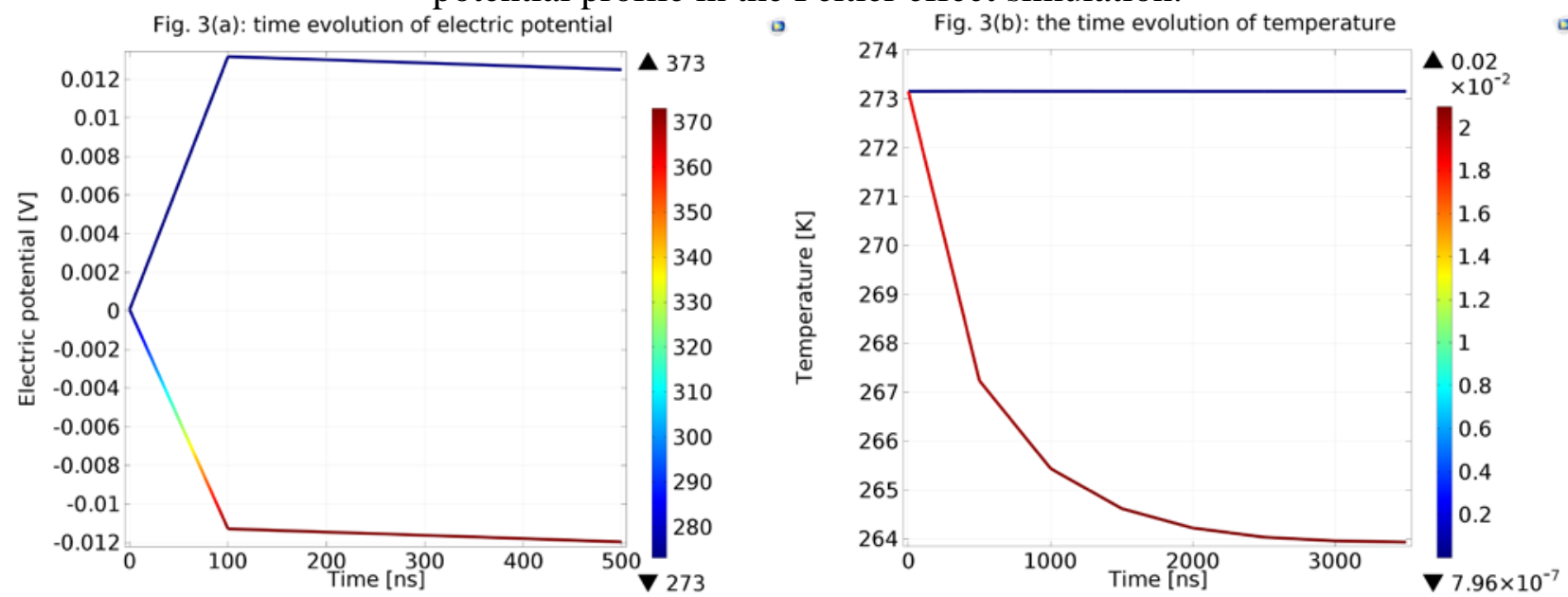

Fig. 3: (a) the time evolution of electric potential measured at each end of the SiNW with color bar served as a temperature meter. (b) the time evolution of temperature measured at each end of the

SiNW with color bar served as a voltmeter

To gain more insight, Fig. 3 demonstrates that the time evolutions of electric potential and temperature at each end of the SiNW, respectively. Simulation results clearly indicates that it takes 
about 100ns to linearly build up the steady state in the Seebeck effect simulation. However, it needs about 500ns to reach the steady state in the Peltier effect simulation. As mentioned before, in the Peltier effect simulation, one end of the SiNW is in contact with a heat sink module, which can keep this end at temperature 273K, which can be seen in Fig. 3(b). The other end of the SiNW is used as current electrode with electric current $1.25 \mathrm{uA}$ through the sample. The electric potential difference of $2 \mathrm{mV}$ between two ends of the SiNW can produce a temperature difference of $9 \mathrm{~K}$ in the Peltier effect simulation.

Finally, Fig. 4 displays electric potential profile versus temperature profiles for the Seebeck effect and the Peltier effect simulations. It is impressive that the Seebeck effect differs the Peltier effect in transient transport behaviors. This means that besides the Seebeck effect and the Peltier effect are the opposite of one another, some other effects, involving a fundamental interplay between the electronic and thermal properties of a thermoelectric system, could play important roles. Certainly, more theoretical explanations and numerical works must be performed before solid conclusions can be drawn.
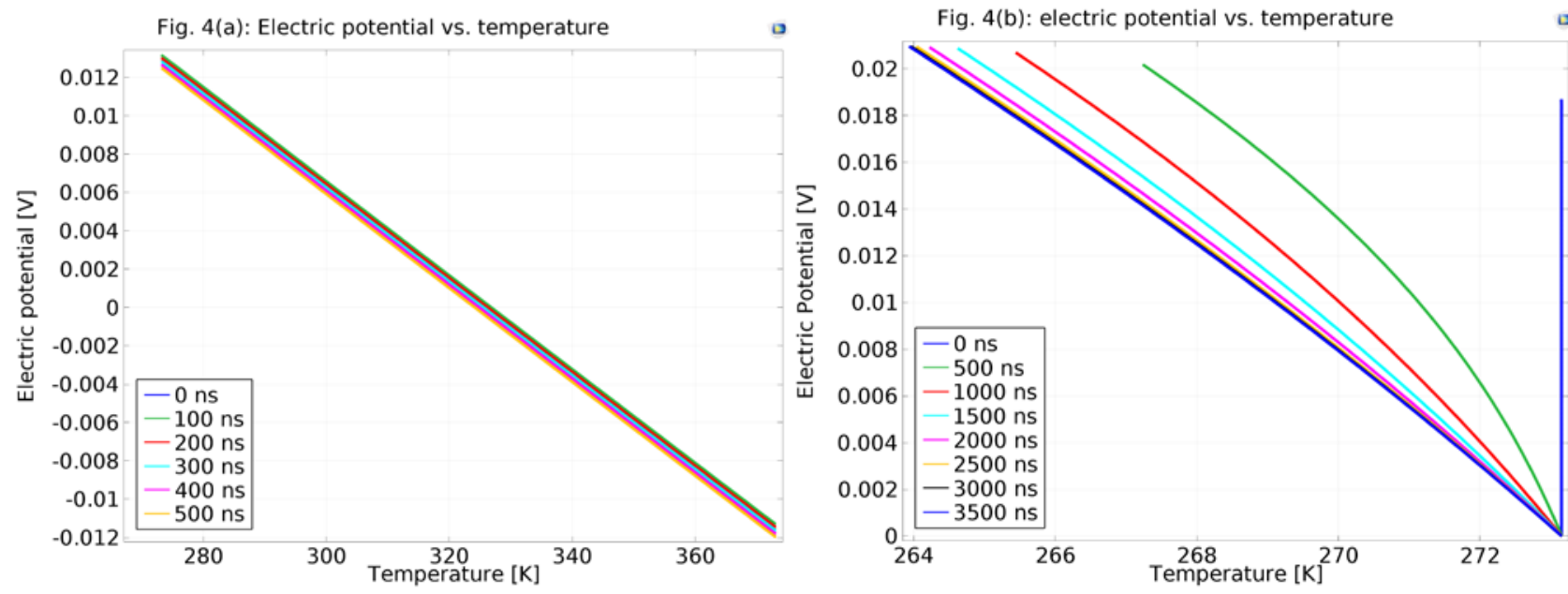

Fig. 4: (a) The Seebeck effect, (b) the Peltier effect.

\section{Conclusion}

Thermoelectric devices are able to transform a temperature gradient into electrical current, and vice versa through simple structures without moving parts. High efficiency thermoelectric materials could promise a great potential for the development of energy harvesting devices. This study have employed finite element analysis using COMSOL Multiphysics to simulate the thermoelectric effects in a SiNW. To demonstrate the transient transport behaviors of the Seebeck effect and Peltier effect, this study have performed these simulations to quantitatively explore the transient behaviors to the electric potential and temperature correspondence under the time-dependent condition. According to the simulations, transient transport appears different with a SiNW which is subjected to a thermal gradient and an electric potential gradient. The Seebeck effect gives rise to a shorter relaxation time of electric flow while the Peltier effect needs more time to build up a steady heat flow. Nevertheless, the laws of thermodynamics can be used to derive all thermoelectric effects, the corresponding transient transports of heat or electric via temperature gradient or electric potential gradient exhibit different in accordance with the simulation.

\section{References}

[1] Goldsmid, H.J. in CRC Handbook of Thermoelectrics (ed. Rowe, D.M.), CRC Press, Boca Raton, FL, 1995.

[2] Hicks L. D., and Dresselhaus M. S., Thermoelectric figure of merit of a one-dimensional conductor [J], Physical Review B, vol. 47, no. 24, June 1993.

[3] Hicks L. D., and Dresselhaus M. S., Effect of quantum-well structures on the thermoelectric 
figure of merit [J], Physical Review B, vol. 47, no.19, May 1993.

[4] Allon I. Hochbaum, Renkun Chen, Raul Diaz Delgado, Wenjie Liang, Erik C. Garnett, Mark Najarian, Arun Majumdar and Peidong Yang, Enhanced thermoelectric performance of rough silicon nanowires [J], Nature Letters, vol. 451, Janurary 2008.

[5] Stranz A., Waag A., and Peiner E., High-temperature performance of stacked silicon nanowires for thermoelectric power generation [J], J. Electron. Mater, vol. 42, no. 7, pp. 2233-2238, Jul. 2013.

[6] Lim J., Hippalgaonkar K., Andrews S. C., Majumdar A., and Yang P,, Quantifying surface roughness effects on phonon transport in silicon nanowires [J], Nano Lett., vol. 12, no. 5, pp. 2475-2482, 2012.

[7] Liang, L. H. \& Li, B., Size-dependent thermal conductivity of nanoscale semiconducting systems [J], Phys. Rev. B, Vol. 73, No. 15, (April 2006) pp. 153303.

[8] Shi L., Yao D., Zhang G., and Li B., Size dependent thermoelectric properties of silicon nanowires [J], Applied Physics Letters, vol. 95, 063102, May 2009.

[9] Zhang G., Zhang Q., Bui C. T., Lo G. Q., and Li B., Thermoelectric performance of silicon nanowires [J], Applied Physics Letters, vol. 94, 213108-1, May 2009.

[10] Bourgeois, O., Fourniew, T. and Chaussy, J., Measurement of the thermal conductance of silicon nanowires at low temperature [J]. Journal of Applied Physics, Vol. 101, No. 1, January 2007, pp. 016104-016106.

[11] Deyu Li, Yiying Wu, Philip Kim, Li Shi, Peidong Yang, and Arun Majumdar, Thermal conductivity of individual silicon nanowires [J], Applied Physics Letters, Vol, 83, No.14, 6 October 2003. 